Supplementary material

\title{
Disproof of the Structure Proposed for a Natural Phenylethanol Isolated from a Mangrove Avicennia marina
}

\author{
Yong-Qing Yang ${ }^{* a, b}$ \\ ${ }^{a}$ School of Pharmacy, Jiangsu University, Zhenjiang, Jiangsu 212013, China; \\ ${ }^{b}$ State Key Laboratory of Bioorganic and Natural Products Chemistry, Shanghai \\ Institute of Organic Chemistry, Chinese Academy of Sciences, 345 Lingling \\ Road, Shanghai 200032, China \\ e-mail: yqy@ujs.edu.cn
}

Table of Contents

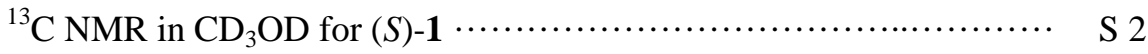

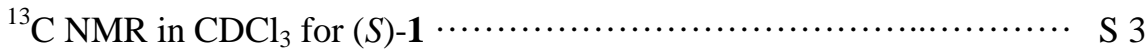

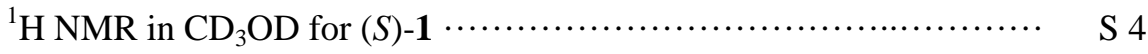

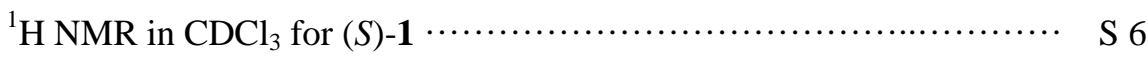

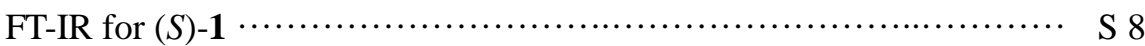

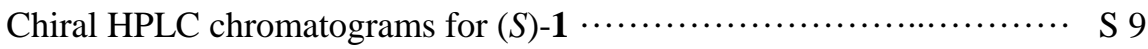

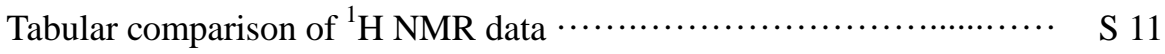

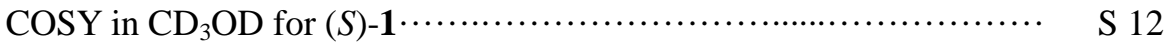

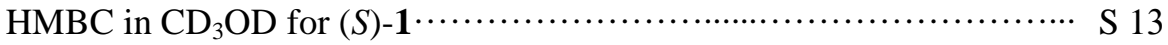

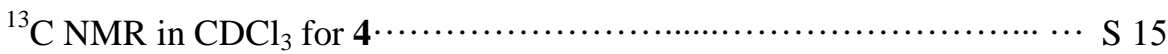

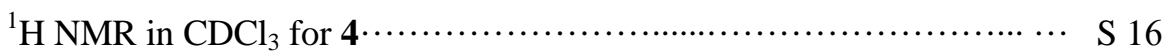

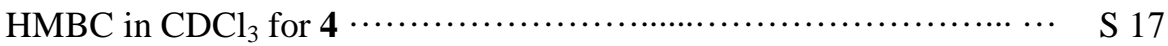


$\angle 00{ }^{\circ} \mathrm{CZ}$

$\downarrow 69^{\circ} 92$

५९ร $6 \varepsilon$

乙งह $8 \mathrm{t}$

$\varepsilon \angle G^{\circ} 8 \mathrm{~V}$

$98 \angle \circ 8$

$0006 t$

OLC 6 t

Ll一 $6 t$

$8 \varepsilon 96 t$

$6 \nabla \nabla \bullet 9$

$\varepsilon 6 \nabla^{\circ} 0 L$

GLL'ZL

$\rightarrow G 9^{\circ} L L$

เ89'9レ

$806^{\circ} 0 \varepsilon L$

งฺt乙Eเ

$\angle \angle 6$ SGL $^{\circ}$

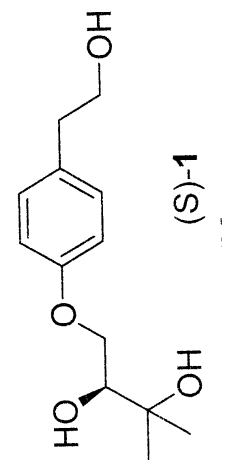


$\varepsilon \varepsilon 0^{\circ} 0^{-}$

$266^{\circ} \nabla z$

$\downarrow 69^{\circ} 92$

Eเ乙 $8 \varepsilon$

6己L\&9

$8 \angle Z 69$

SOLLL

$\forall \forall 9^{\circ} G L$

$8 \varepsilon L 9 L$

$000 \angle L$

$9 t Z \angle L$

98น๖レレ

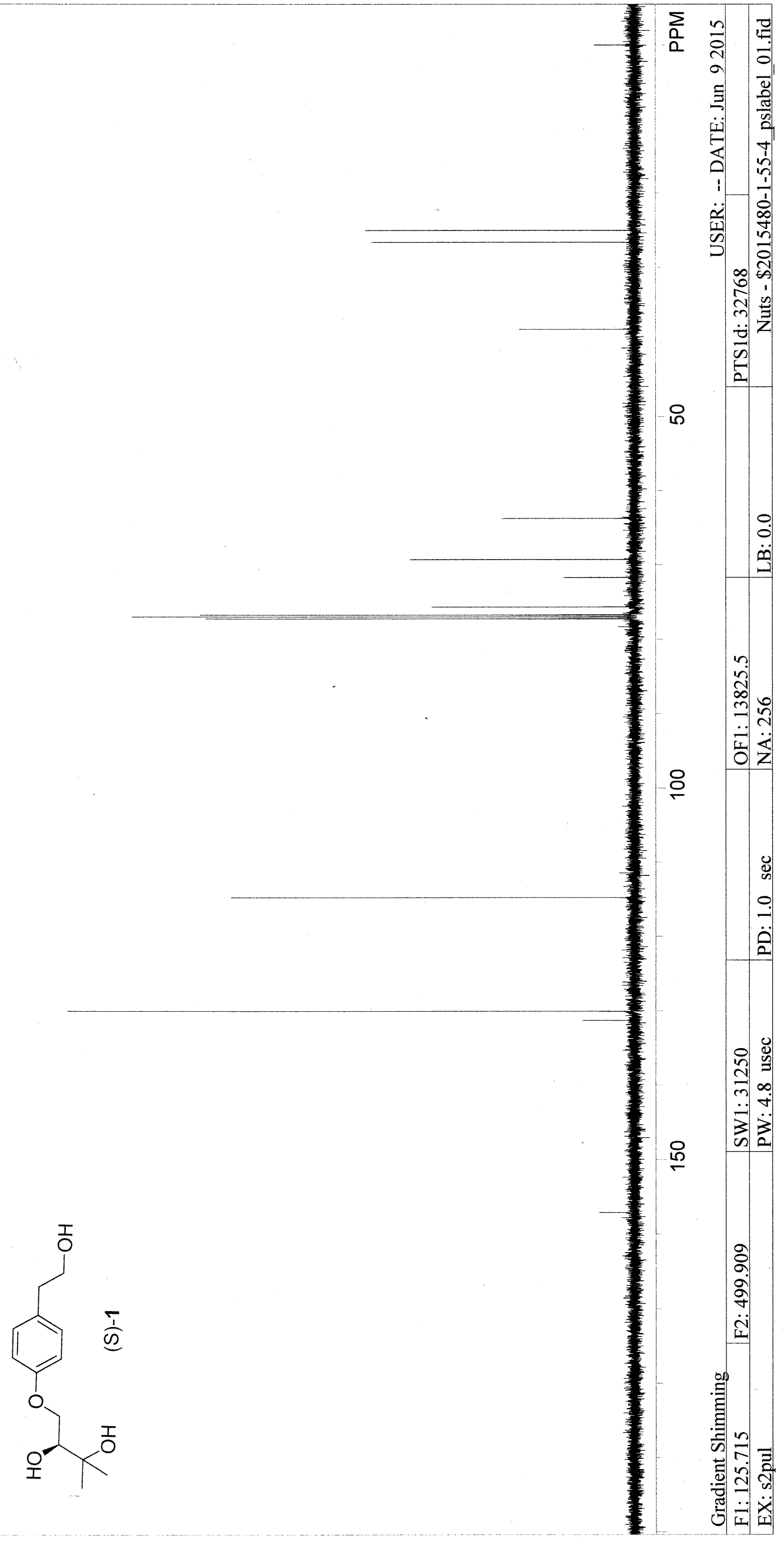

เ80 $0 \varepsilon$

$60 \varepsilon^{\prime} L E L$

$890^{\circ}$ LGL 

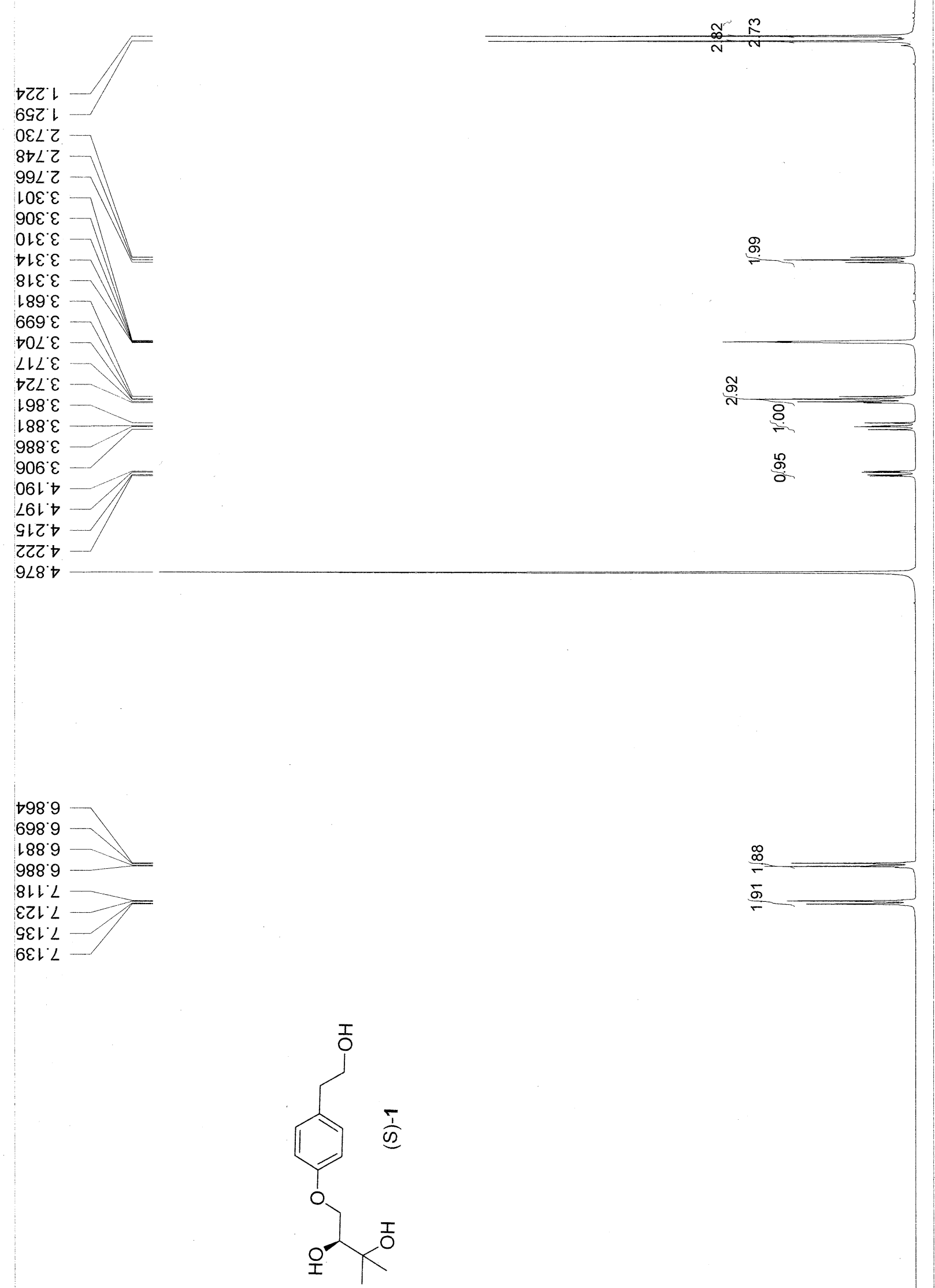

0

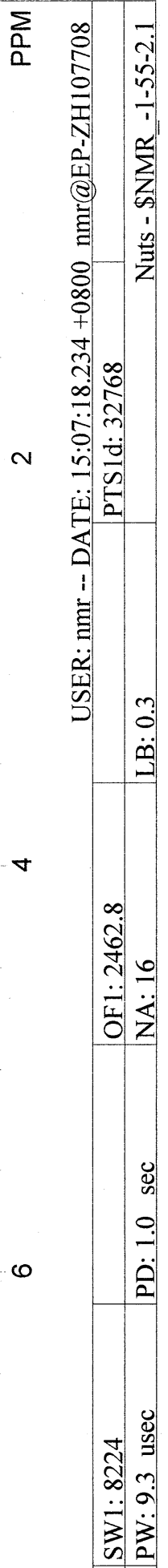

$\infty$

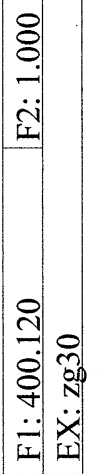




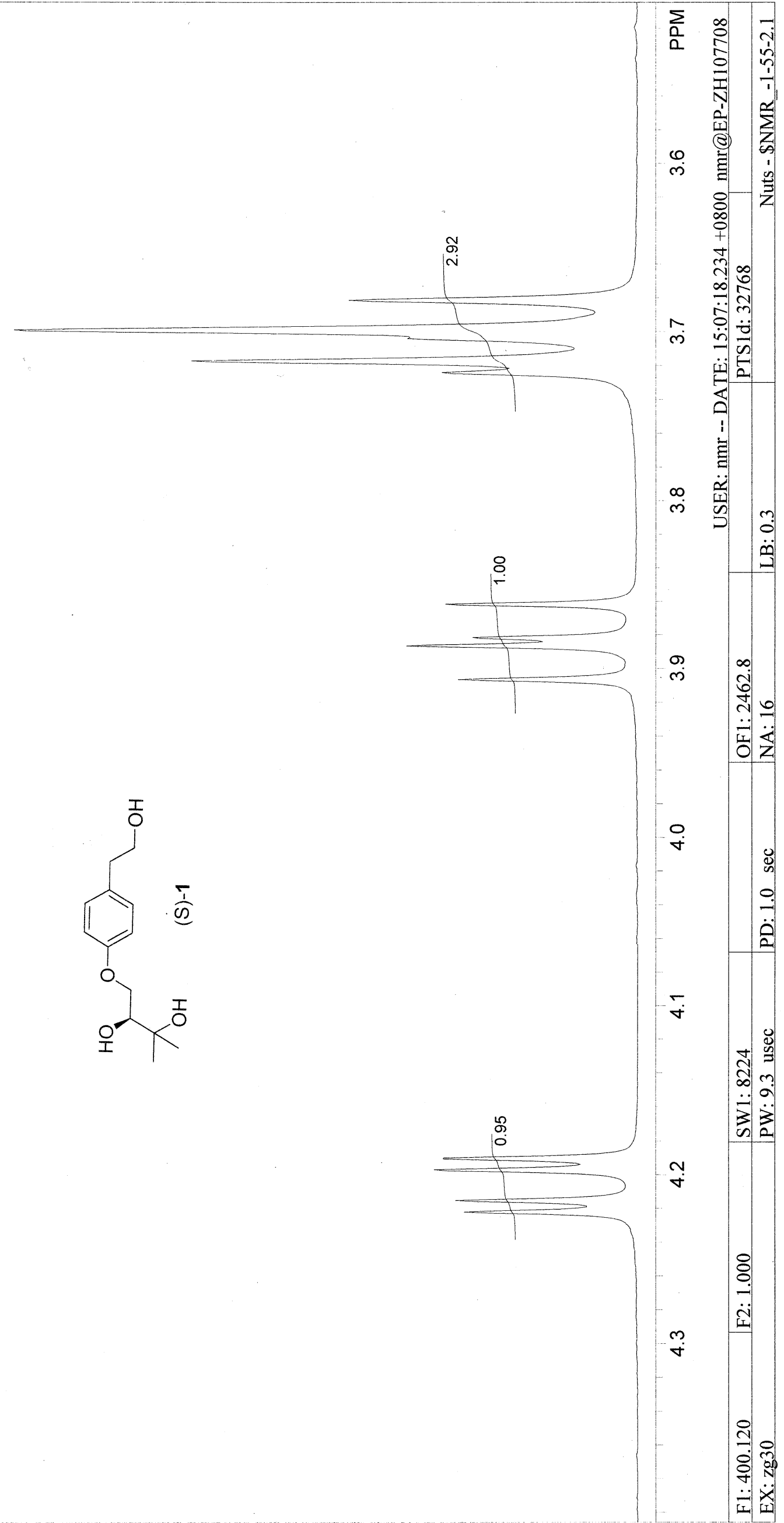



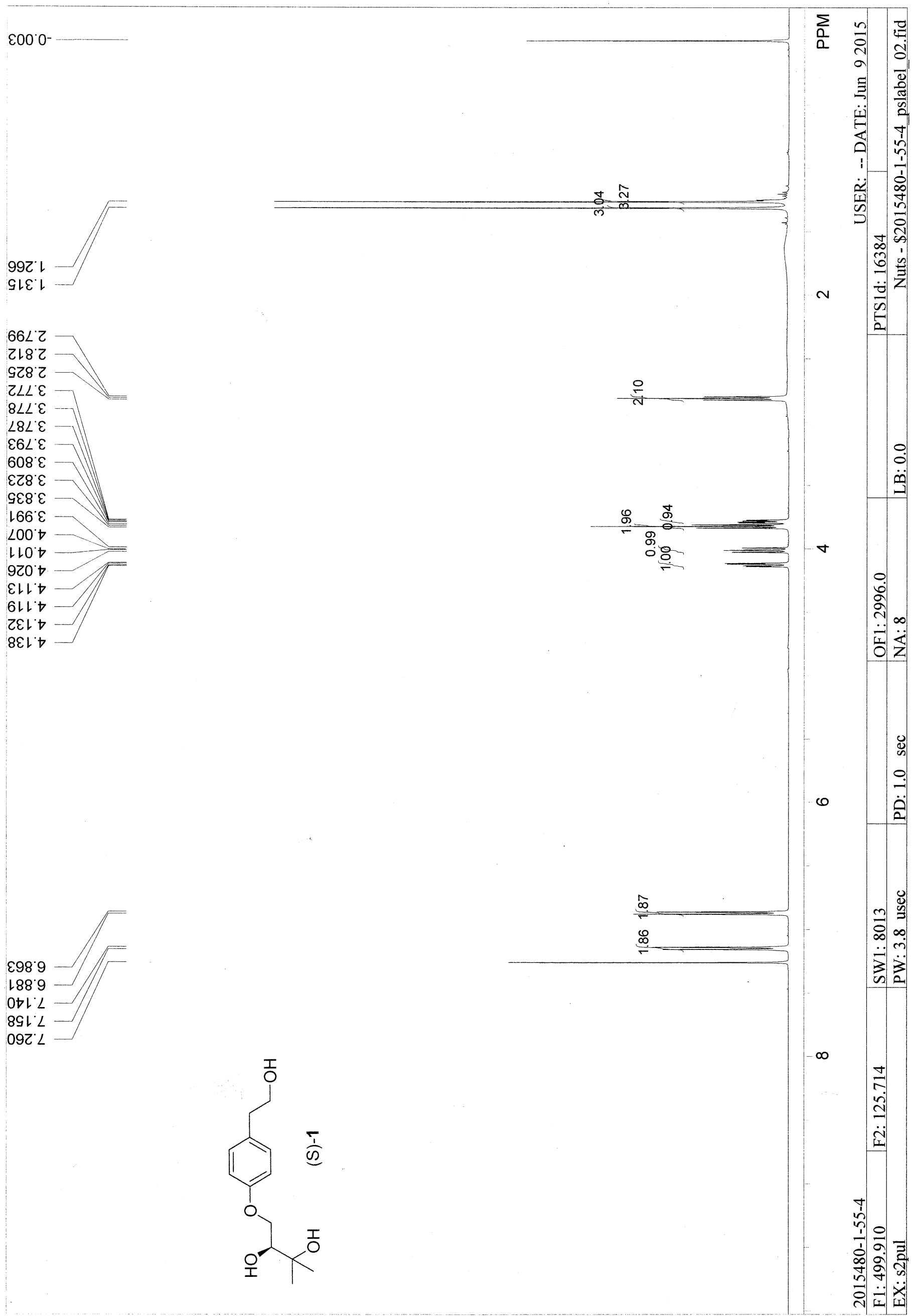

$66 L 2$

2 2182

$928 \mathrm{Z}$

ZLL'

$8 \angle L ' \mathcal{B}$

$\angle 8 \angle \mathcal{L} E$

$\varepsilon 6 L^{\circ} \varepsilon$

$608^{\circ} \varepsilon$

$\varepsilon)^{\circ} \varepsilon$

५ร8 $\varepsilon$

$166^{\circ} \varepsilon$

$\angle 00^{\circ} \mathrm{O}$

LLO

$920 \%$

ELt

6Lt'

ZEL

$8 \varepsilon+5$

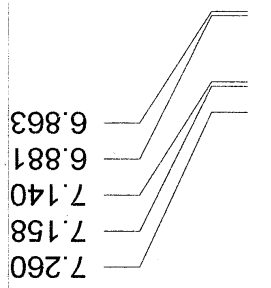



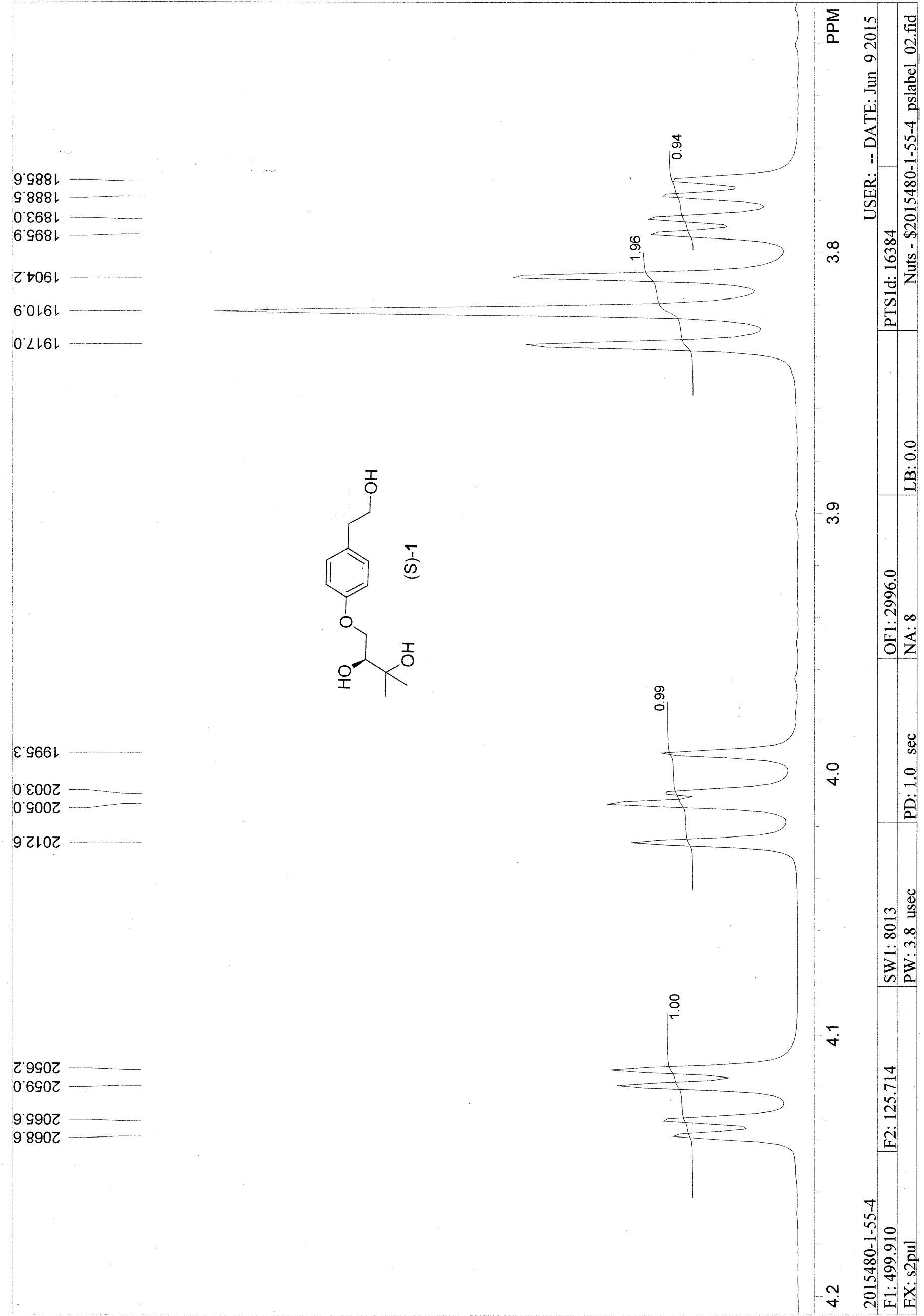


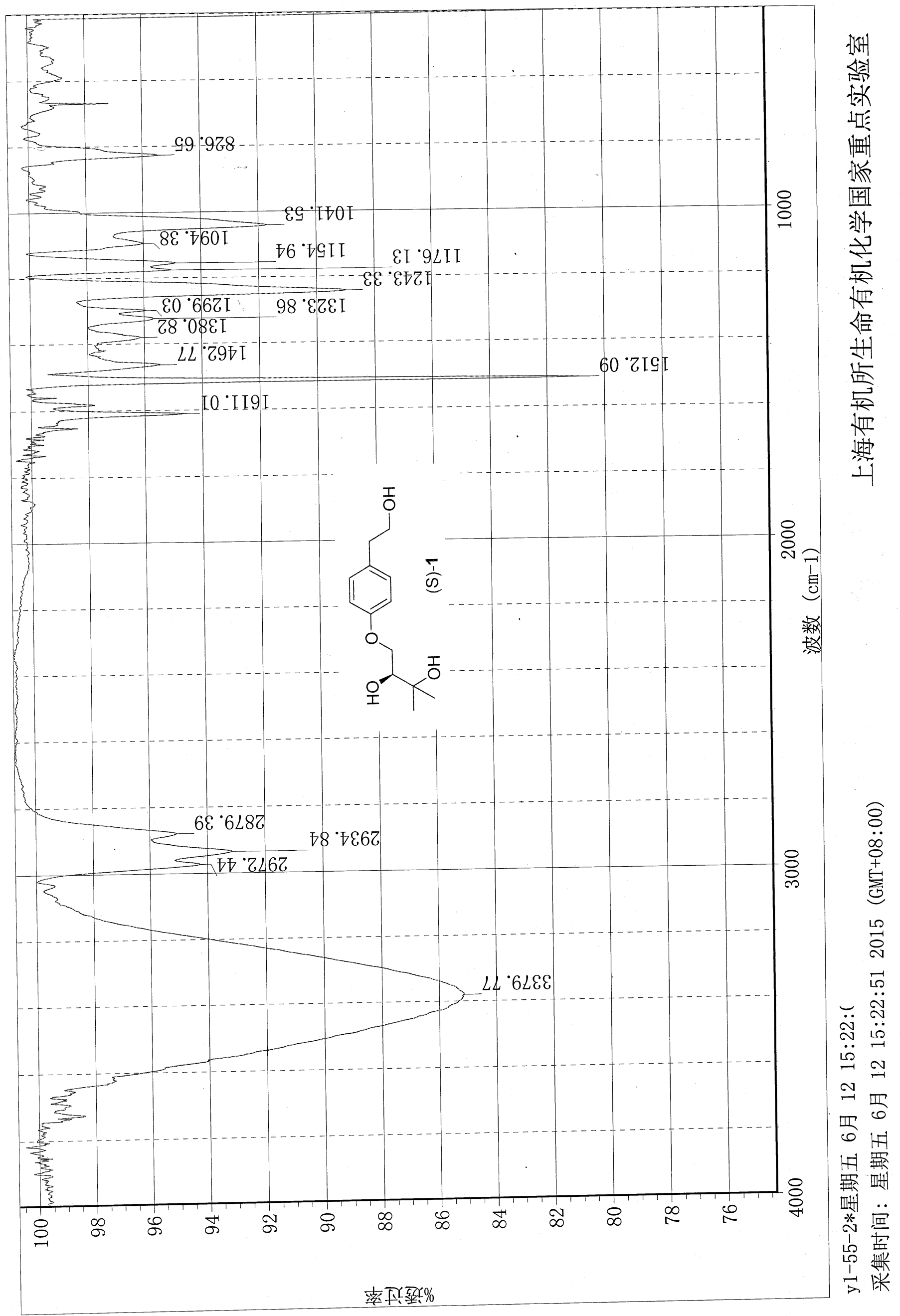




\section{YYQ-1-55-2 PA-2 552140.7}

\begin{tabular}{llll} 
Sample Name: & YYQ-1-55-2 PA-2 55 214 0.7 & Injection Volume: & $\mathbf{3 . 0}$ \\
Vial Number: & BC8 & Channel: & UV_VIS_2 \\
Sample Type: & unknown & Wavelength: & $\mathbf{2 1 4 . 0}$ \\
Control Program: & test-dad3 & Bandwidth: & $\mathbf{4}$ \\
Quantif. Method: & WXL & Dilution Factor: & $\mathbf{1 . 0 0 0 0}$ \\
Recording Time: & $\mathbf{2 0 1 5 - 7 - 1 3 ~ 1 4 : 5 8}$ & Sample Weight: & $\mathbf{1 . 0 0 0 0}$ \\
Run Time (min): & $\mathbf{1 7 . 5 1}$ & Sample Amount: & $\mathbf{1 . 0 0 0 0}$ \\
\hline
\end{tabular}

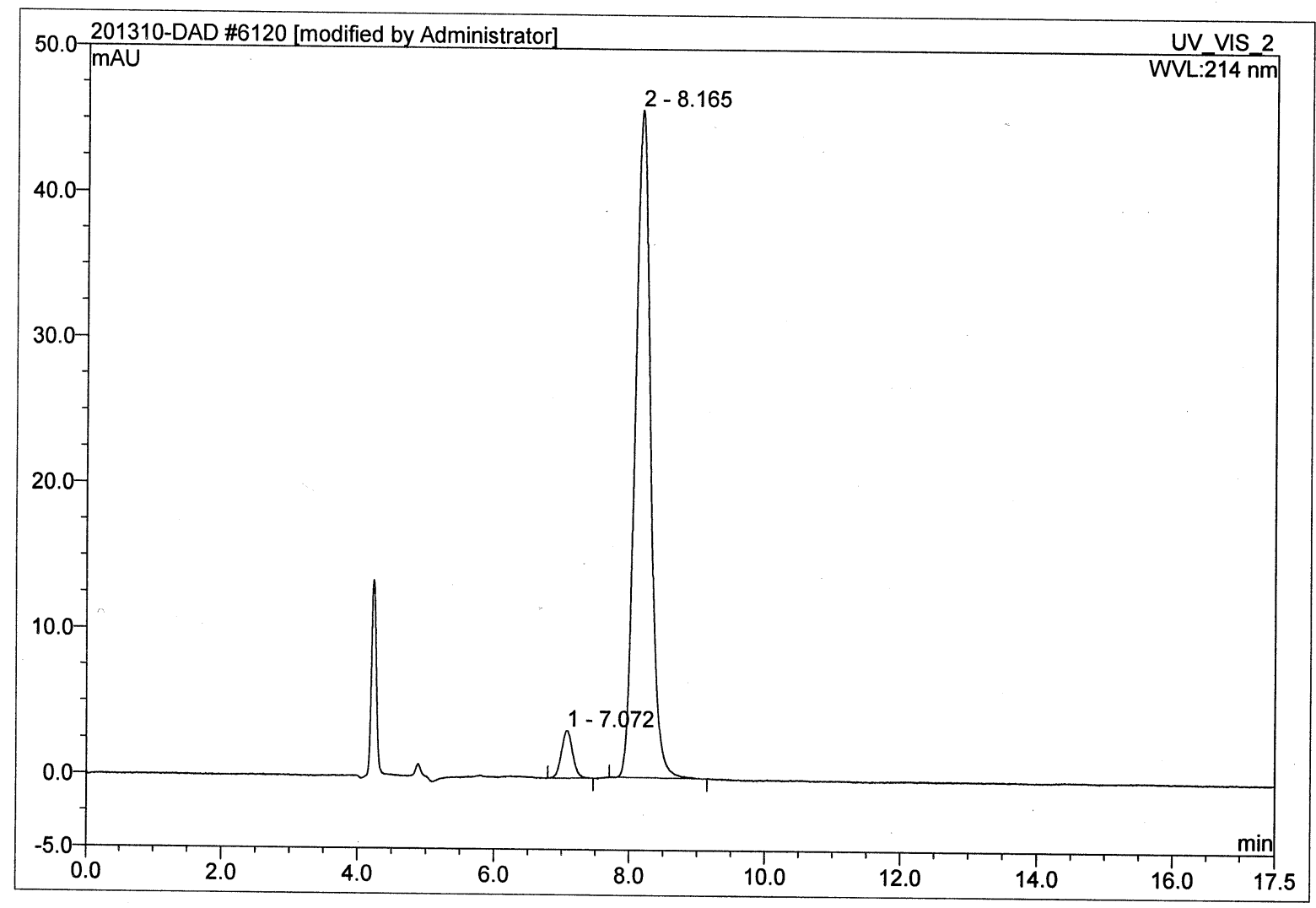

\begin{tabular}{|c|ccrrrrr|}
\hline No. & $\begin{array}{c}\text { Ret.Time } \\
\text { min }\end{array}$ & Peak Name & $\begin{array}{c}\text { Height } \\
\text { mAU }\end{array}$ & $\begin{array}{c}\text { Area } \\
\text { mAU*min }\end{array}$ & $\begin{array}{r}\text { Rel.Area } \\
\%\end{array}$ & Amount & Type \\
\hline 1 & 7.07 & n.a. & 3.269 & 0.622 & 4.94 & n.a. & BMB $^{*}$ \\
2 & 8.17 & n.a. & 45.840 & 11.959 & 95.06 & n.a. & BMB $^{*}$ \\
\hline Total: & & & 49.110 & 12.581 & 100.00 & 0.000 & \\
\hline
\end{tabular}

Chiral HPLC analysis of $(S)-1$ 


\begin{tabular}{|llll|}
\hline 6119 YYQ-1-69-2+- PA-2 552140.7 & & \\
& & & \\
\hline Sample Name: & YYQ-1-69-2+- PA-2 55214 & & \\
Vial Number: & BD8 & Injection Volume: & $\mathbf{6 . 0}$ \\
Sample Type: & unknown & Channel: & UV_VIS_2 \\
Control Program: & test-dad3 & Wavelength: & $\mathbf{2 1 4 . 0}$ \\
Quantif. Method: & WXL & Bandwidth: & $\mathbf{4}$ \\
Recording Time: & $\mathbf{2 0 1 5 - 7 - 1 3 ~ 1 3 : 4 5}$ & Dilution Factor: & 1.0000 \\
Run Time (min): & $\mathbf{7 1 . 7 1}$ & Sample Weight: & $\mathbf{1 . 0 0 0 0}$ \\
\end{tabular}

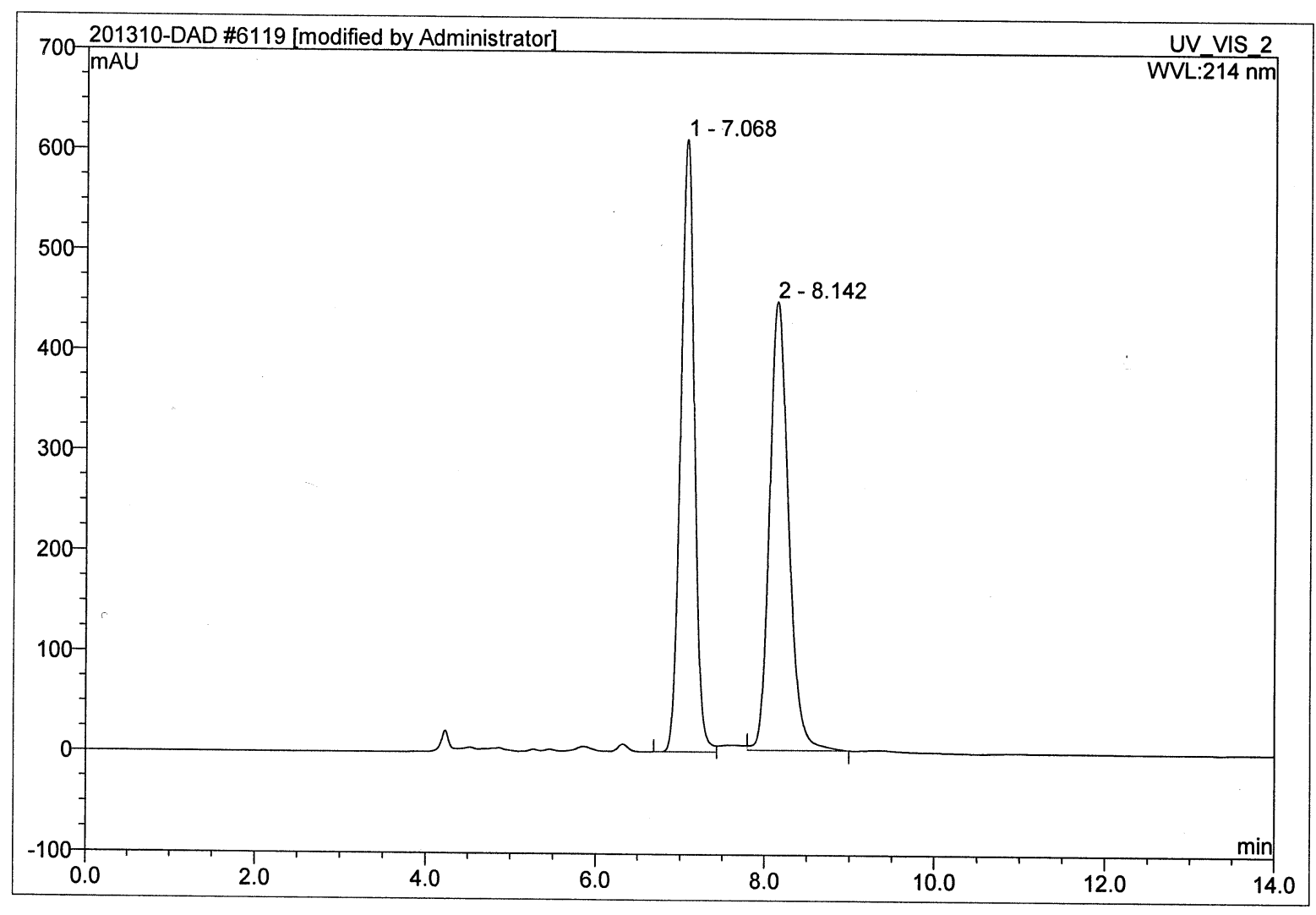

\begin{tabular}{|r|ccccccc|}
\hline No. & $\begin{array}{c}\text { Ret.Time } \\
\text { min }\end{array}$ & Peak Name & $\begin{array}{c}\text { Height } \\
\text { mAU }\end{array}$ & $\begin{array}{c}\text { Area } \\
\text { mAU*min }\end{array}$ & $\begin{array}{r}\text { Rel.Area } \\
\%\end{array}$ & Amount & Type \\
\hline 1 & 7.07 & n.a. & 610.617 & 115.217 & 49.56 & n.a. & BM $^{*}$ \\
2 & 8.14 & n.a. & 447.814 & 117.280 & 50.44 & n.a. & MB $^{*}$ \\
\hline Total: & & & 1058.432 & 232.497 & 100.00 & 0.000 & \\
\hline
\end{tabular}

\section{Chiral HPLC analysis of racemic 1}




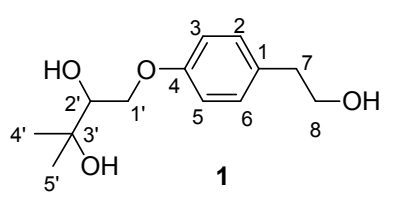

Table S-1. Comparison of ${ }^{1} \mathrm{H}$ NMR for the natural and synthetic 1

\begin{tabular}{|l|l|l|}
\hline Natural $\mathbf{1}\left(500 \mathrm{MHz}, \mathrm{CD}_{3} \mathrm{OD}\right)^{a}$ & Synthetic $\mathbf{1}\left(400 \mathrm{MHz}, \mathrm{CD}_{3} \mathrm{OD}\right)^{b}$ & Synthetic $1\left(500 \mathrm{MHz}, \mathrm{CDCl}_{3}\right)^{c}$ \\
\hline $7.14(\mathrm{~d}, J=7.5 \mathrm{~Hz}, 2 \mathrm{H}, \mathrm{H}-2,6)$ & $7.13(\mathrm{~d}, J=8.8 \mathrm{~Hz}, 2 \mathrm{H})$ & $7.15(\mathrm{~d}, J=9.0 \mathrm{~Hz}, 2 \mathrm{H})$ \\
\hline $6.89(\mathrm{~d}, J=7.5 \mathrm{~Hz}, 2 \mathrm{H}, \mathrm{H}-3,5)$ & $6.88(\mathrm{~d}, J=8.8 \mathrm{~Hz}, 2 \mathrm{H})$ & $6.87(\mathrm{~d}, J=9.0 \mathrm{~Hz}, 2 \mathrm{H})$ \\
\hline $4.23\left(\mathrm{~d}, J=9.3 \mathrm{~Hz}, 1 \mathrm{H}, \mathrm{H}-1^{\prime}\right)$ & $4.21(\mathrm{dd}, J=10,2.8 \mathrm{~Hz}, 1 \mathrm{H})$ & $4.13(\mathrm{dd}, J=9.5,3.0 \mathrm{~Hz}, 1 \mathrm{H})$ \\
\hline $3.89\left(\mathrm{~d}, J=9.3,1 \mathrm{H}, \mathrm{H}-1^{\prime}\right)$ & $3.88(\mathrm{dd}, J=10,8.0 \mathrm{~Hz}, 1 \mathrm{H})$ & $4.01(\mathrm{dd}, J=9.5,7.5 \mathrm{~Hz}, 1 \mathrm{H})$ \\
\hline $3.75\left(\mathrm{br}, 1 \mathrm{H}, \mathrm{H}-2^{\prime}\right)$ & $3.72-3.68(\mathrm{~m}, 1 \mathrm{H})$ & $3.82(\mathrm{t}, J=6.5 \mathrm{~Hz}, 2 \mathrm{H})$ \\
\hline $3.69(\mathrm{t}, J=7.2,2 \mathrm{H}, \mathrm{H}-8)$ & $3.70(\mathrm{t}, J=7.2 \mathrm{~Hz}, 2 \mathrm{H})$ & $3.78(\mathrm{dd}, J=7.5,3.0 \mathrm{~Hz}, 1 \mathrm{H})$ \\
\hline $2.72(\mathrm{t}, J=7.2 \mathrm{~Hz}, 2 \mathrm{H}, \mathrm{H}-7)$ & $2.75(\mathrm{t}, J=6.8 \mathrm{~Hz}, 2 \mathrm{H})$ & $2.81(\mathrm{t}, J=6.5 \mathrm{~Hz}, 2 \mathrm{H})$ \\
\hline $1.27\left(\mathrm{~s}, 3 \mathrm{H}, \mathrm{H}-4^{\prime}\right)$ & $1.26(\mathrm{~s}, 3 \mathrm{H})$ & $1.32(\mathrm{~s}, 3 \mathrm{H})$ \\
\hline $1.23\left(\mathrm{~s}, 3 \mathrm{H}, \mathrm{H}-5^{\prime}\right)$ & $1.23(\mathrm{~s}, 3 \mathrm{H})$ & $1.27(\mathrm{~s}, 3 \mathrm{H})$ \\
\hline
\end{tabular}

${ }^{a}$ Taken with assignments from (Zheng et al. 2014). ${ }^{b}$ This work.

Zheng, C.; Chen, Y.; Jiang, L. L.; Shi, X.M., 2014. Antiproliferative metabolites from the endophytic fungus Penicillium sp. FJ-1 isolated from a mangrove Avicennia marina. Phytochem. Lett. 10, 272-275. 


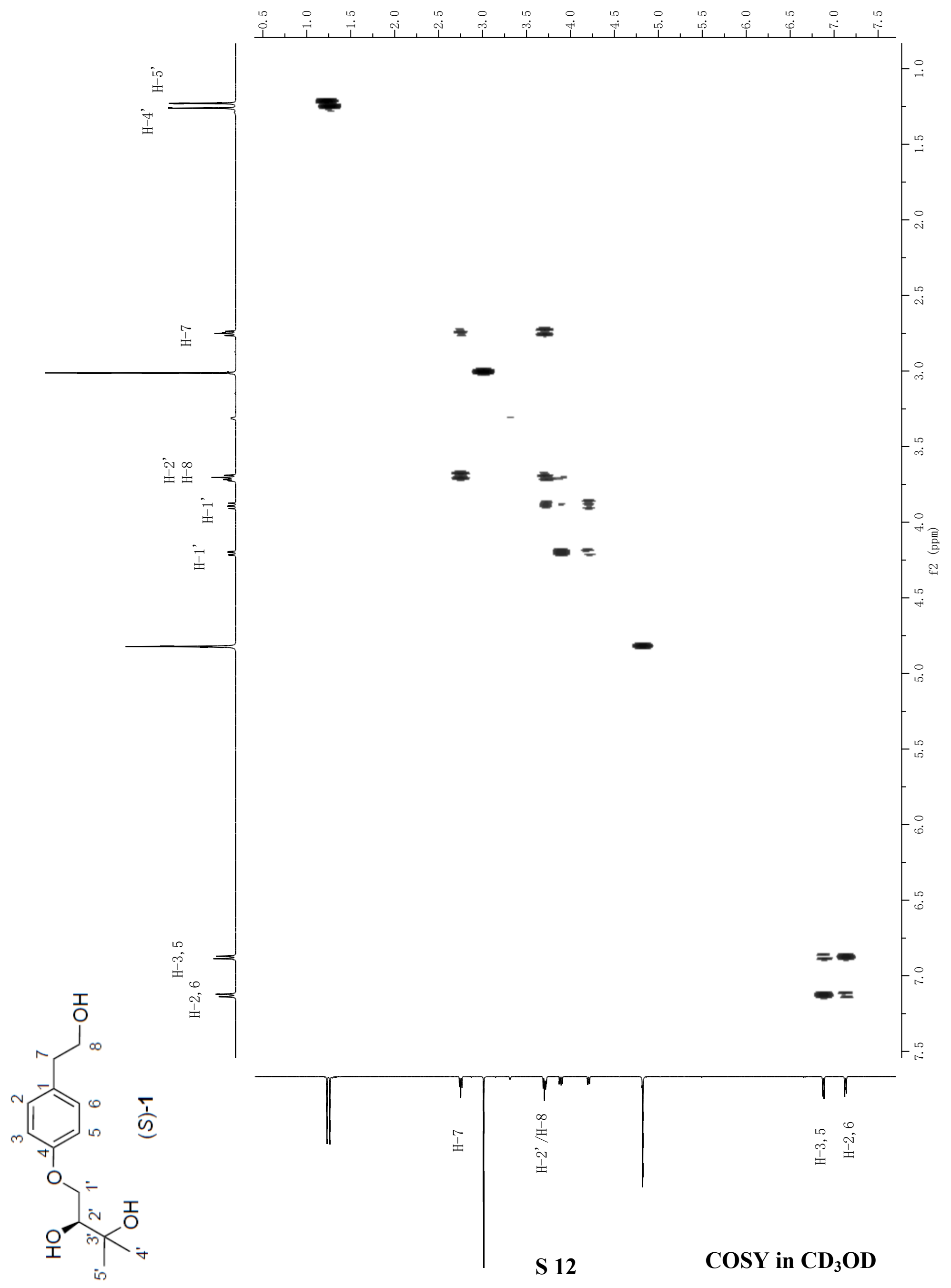




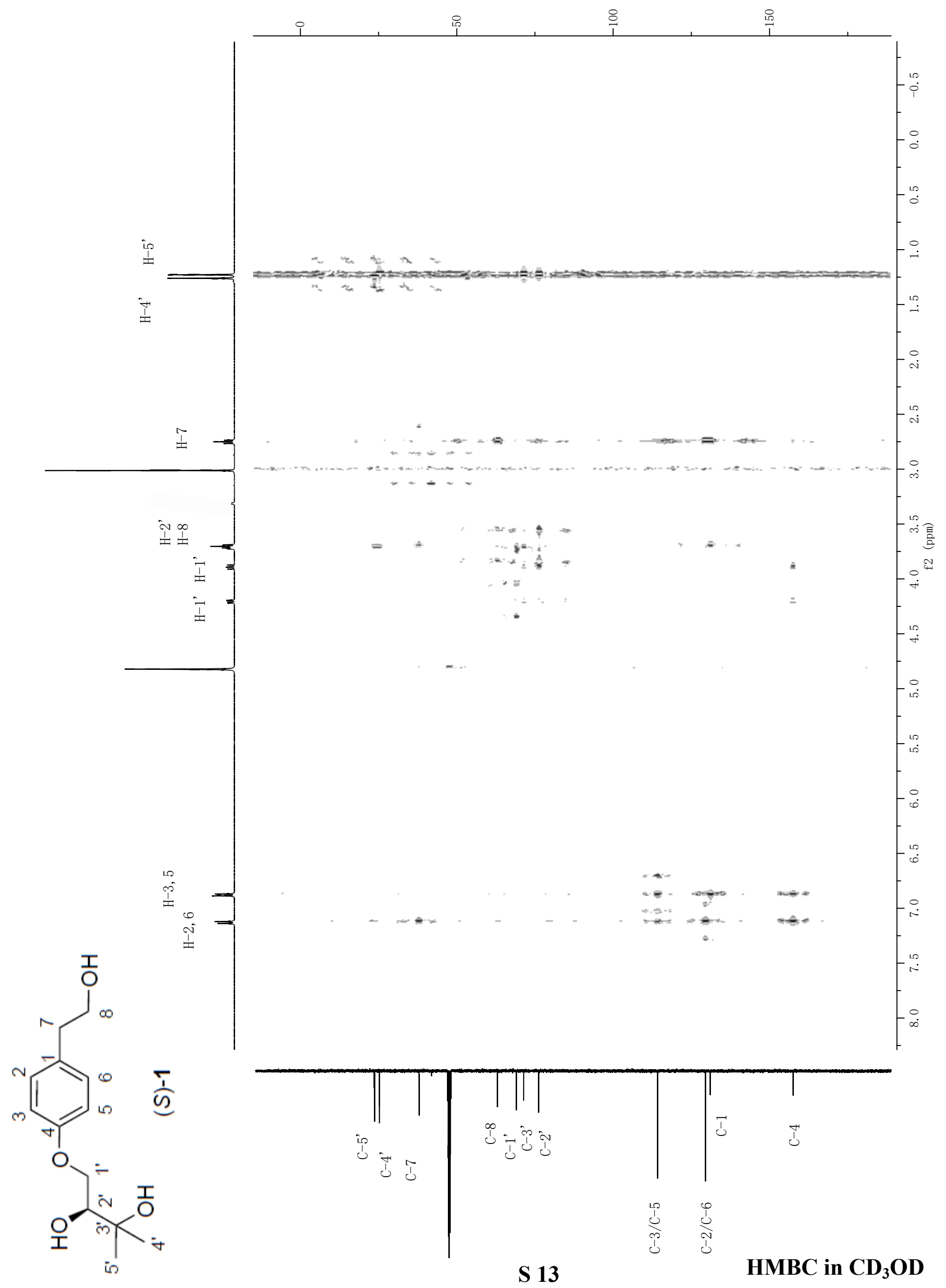




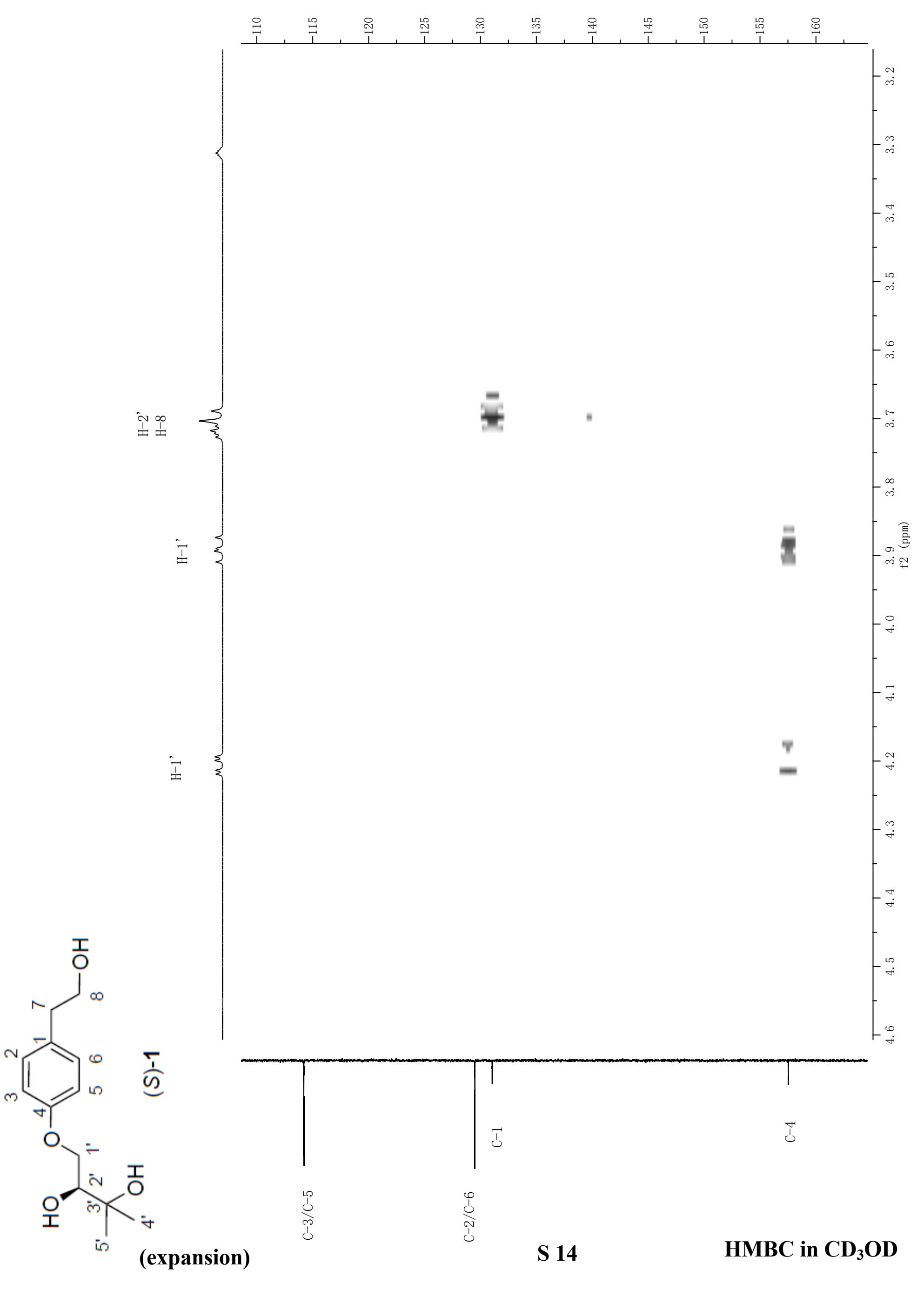


$8700^{\circ}$

$8 \varepsilon L^{\circ} 81$

98L'G

$\varepsilon \succ{ }^{\circ} 8 \varepsilon$

08L'\&9

$8 \varepsilon L ' t 9$

8†L $9 L$

$000^{\circ} \angle L$

$99{ }^{\prime} \angle L$

99よ゚レ

8ZL6レ

S68.62L

68Z $0 \varepsilon\llcorner$

$\varepsilon \angle 0^{\circ} 8 \varepsilon$

6EG' $\angle \mathcal{L}$

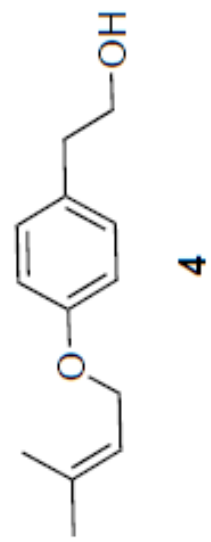


ItIL

09Z L

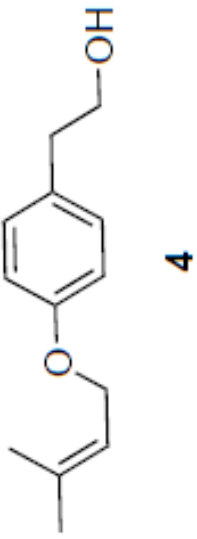




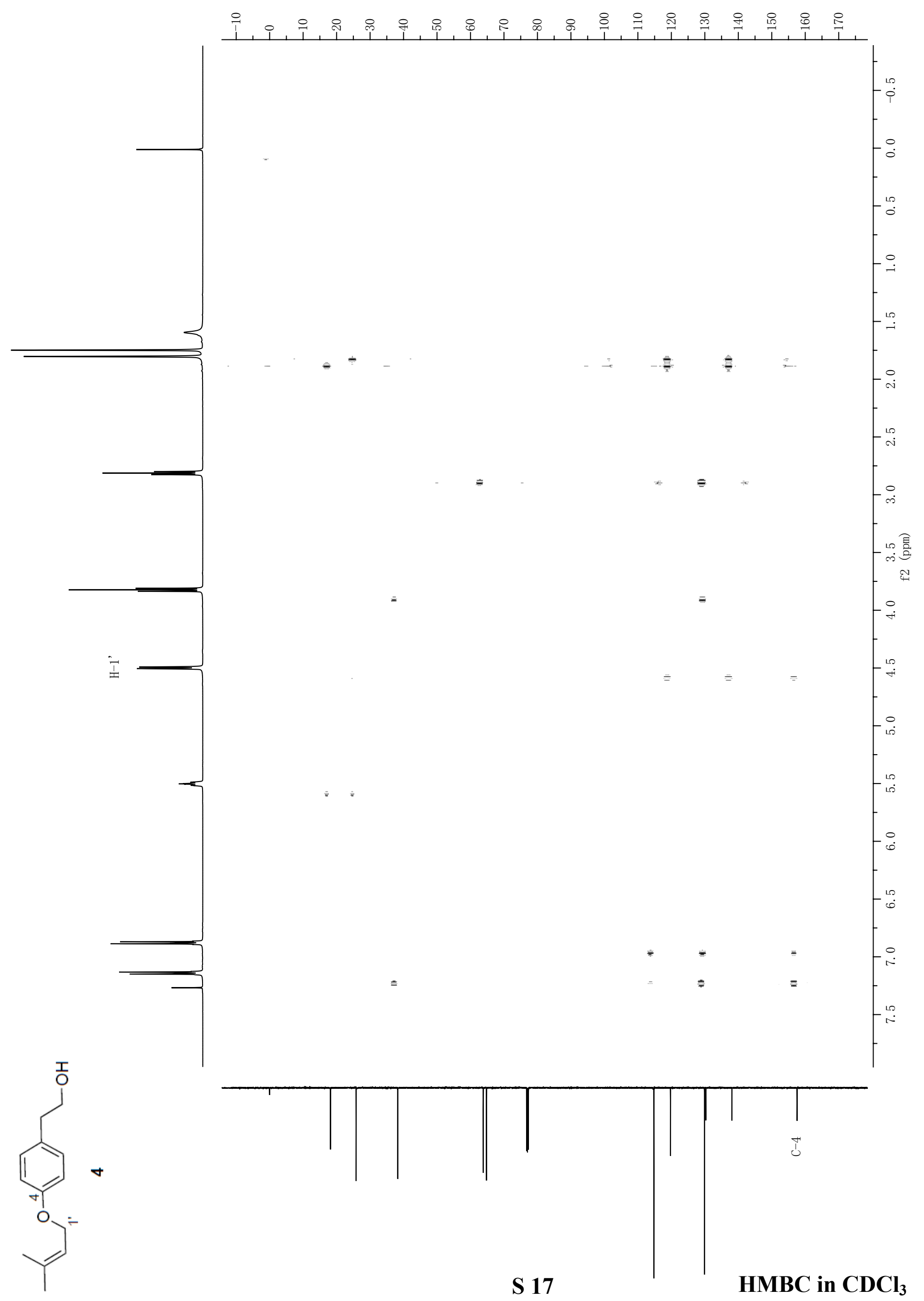


(wdd) If

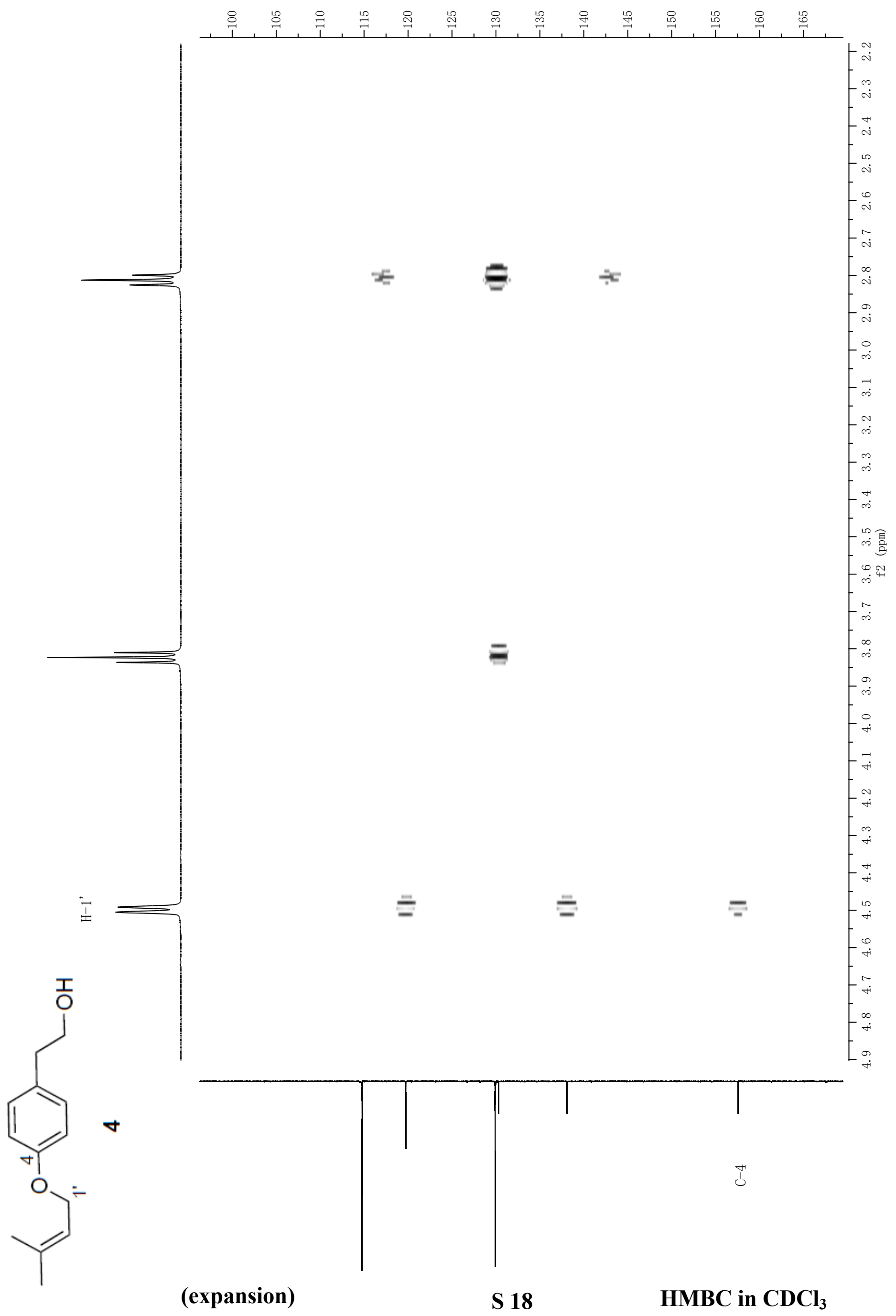

